TRANSACTIONS OF THE

AMERICAN MATHEMATICAL SOCIETY

Volume 348, Number 8, August 1996

\title{
ON VANISHING OF CHARACTERISTIC NUMBERS IN POINCARÉ COMPLEXES
}

\author{
YANGHYUN BYUN
}

\begin{abstract}
Let $G_{r}(X) \subset \pi_{r}(X)$ be the evaluation subgroup as defined by Gottlieb. Assume the Hurewicz map $G_{r}(X) \rightarrow H_{r}(X ; R)$ is non-trivial and $R$ is a field. We will prove: if $X$ is a Poincaré complex oriented in $R$-coefficient, all the characteristic numbers of $X$ in $R$-coefficient vanish. Similarly, if $R=Z_{p}$ and $X$ is a $Z_{p}$-Poincaré complex, then all the $\bmod p$ Wu numbers vanish. We will also show that the existence of a non-trivial derivation on $H^{*}\left(X ; Z_{p}\right)$ with some suitable conditions implies vanishing of $\bmod p \mathrm{Wu}$ numbers.
\end{abstract}

\section{INTRODUCTION}

D. H. Gottlieb proved the following interesting fact ([3]): If $X$ is a connected finite aspherical complex and $Z\left(\pi_{1}(X)\right) \neq 1$, then $\chi(X)=0$. Here $Z(G)$ means the center for any group $G$ and $\chi(X)$ is the Euler-Poincaré number of $X$. This paper is largely motivated by the question of whether the condition $Z\left(\pi_{1}(X)\right) \neq 1$ also implies the vanishing of the Stiefel-Whitney numbers if $X$ is a compact closed aspherical manifold. The question is only partially answered, but in a more general setting.

The paper is mainly about the relation between the characteristic numbers of a Poincaré complex $X$ and its evaluation subgroup, which is the subgroup $G_{r}\left(X, x_{0}\right)$ of $\pi_{r}\left(X, x_{0}\right), x_{0}$ being a base point of $X$, defined as follows by Gottlieb ([4]):

Definition. Let $S^{r}$ denote the $r$-sphere. Consider a continuous function

$$
F: S^{r} \times X \rightarrow X
$$

such that $F\left(z_{0}, x\right)=x$, where $x \in X$ and $z_{0}$ is a basepoint of $S^{r}$. Then $G_{r}\left(X, x_{0}\right)$ consists of all the elements in $\pi_{r}\left(X, x_{0}\right)$ represented by the map $f:\left(S^{r}, z_{0}\right) \rightarrow$ $\left(X, x_{0}\right)$ defined by $f(z)=F\left(z, x_{0}\right)$ for some $F$ such as the above.

Let $R$ be a commutative ring with a unit. Then, by an $n$-dimensional $R$-Poincaré complex, we will mean a CW complex $X$ for which there is a homology class $[X] \in$ $H_{n}(X ; R)$ such that the map $\bigcap[X]: H^{*}(X ; R) \rightarrow H_{n-*}(X ; R)$ is an isomorphism.

In sections 2 and 3 , we will deal with $Z_{p}$-Poincaré complexes $X$ for any prime integer $p$. Then some mod $p$ characteristic numbers of $X$ can be defined using the mod $p$ Steenrod powers as in section 2 without referring to any vector bundle or spherical fibration. We call them the $\bmod p W u$ numbers of $X$. In particular, when

Received by the editors November 14, 1994 and, in revised form, March 20, 1995.

1991 Mathematics Subject Classification. Primary 57P10, 57N65.

Key words and phrases. Characteristic numbers, evaluation subgroup, Hurewicz map.

(C)1996 American Mathematical Society 
$p=2$ and $X$ is a manifold, these characteristic numbers are none other than the usual Stiefel-Whitney numbers of $X$.

The following answers the question asked above:

Theorem 1. Let $p$ be a prime and $X$ a connected aspherical $Z_{p}$-Poincaré complex. If there is an element in $Z\left(\pi_{1}(X)\right)$ whose image in $H_{1}\left(X ; Z_{p}\right)$ under the Hurewicz map is not zero, then all the mod $p$ Wu numbers of $X$ vanish.

Note that more than just $Z\left(\pi_{1}(X)\right) \neq 1$ is required in the above for the $\bmod p$ Wu numbers to vanish. Therefore a question can be raised, for which the author does not know the answer at the moment: Is there an example of a connected aspherical $Z_{p}$-Poincaré complex $X$ such that $Z\left(\pi_{1}(X)\right) \neq 1$ but some of the mod $p$ Wu numbers are nonzero?

Definition. Let $X$ be a space and $R$ a commutative ring with identity. A derivation of degree $-r$ on $H^{*}(X ; R)$ is a group homomorphism $D: H^{*}(X ; R) \rightarrow H^{*-r}(X ; R)$ such that $D(u \cup v)=D u \cup v+(-1)^{r|u|} u \cup D v$ for any $u, v \in H^{*}(X)$. Here $|u|$ means dim $u$.

On the way to proving Theorem 1, we will establish the following lemma which relates the derivation to the vanishing of the $\bmod p \mathrm{Wu}$ numbers:

Lemma 2. Let $X$ be a $Z_{p}$-Poincaré space for a prime $p$. Assume there is a derivation $D$ of deg $-r, r>0$, on $H^{*}(X)$ which commutes with the mod $p$ Steenrod powers. Also assume there is a class $\varphi \in H^{r}(X)$ such that $D \varphi=1, \wp^{i} \varphi=0$ for any $i$ such that $0<i<r$ if $p=2$ and $0<i<[r / 2]$ if $p>2$, where the $\wp^{i}$ 's are the mod $p i$-th Steenrod powers. Then all the mod $p$ Wu numbers of $X$ vanish.

In particular, the existence of a nontrivial derivation of deg -1 commuting with the mod $p$ Steenrod powers on a $Z_{p}$-Poincaré complex implies vanishing of all of its $\bmod p \mathrm{Wu}$ numbers.

This lemma will be directly applied to projective planes to give a succinct argument to decide which of them are boundaries (see the Remark that follows the proof in section 3).

In section 4, we will deal the same issue in a more geometric way, confining ourselves to the Poincaré complexes in the sense of Wall([15]), in which case the characteristic numbers of $X$ come from its Spivak normal fibration (see the definition and the comments following it, before the proof of Theorem 3). We will prove:

Theorem 3. Let $M$ be a connected Poincaré complex of formal dimension n, oriented with respect to a field coefficient $R$. Assume there is an $\alpha \in G_{k}\left(M, x_{0}\right)$ such that $h_{R}(\alpha) \neq 0$, where $h_{R}: \pi_{k}\left(M, x_{0}\right) \rightarrow H_{k}(M ; R)$ is the Hurewicz homomorphism and $x_{0}$ is a base point. Then all the characteristic numbers in $R$-coefficients coming from the Spivak fibration vanish.

The author wishes to express his deep gratitude to Professor D. H. Gottlieb for the outline of the argument in section 4 . The author also would like to thank his advisor, Professor Frank X. Connolly, for suggesting the problem, and for helpful conversations and encouragement. 


\section{The mod $p$ Wu numbers of $Z_{p}$-Poincaré spaces}

From now on, to simplify some statements, we will denote the $k$-th Steenrod square by $\wp^{k}$, specifying $Z_{2}$ coefficients if necessary, even though it is customary to denote it by $S q^{k}$.

Let $p$ be a prime and assume $\xi$ is a sphere fibration over $X$ orientable in $Z_{p^{-}}$ coefficients. Then the mod $p k$-th Wu class $q_{k}(\xi)$ is defined as follows ([9]):

$$
q_{k}(\xi)=\Phi^{-1} \wp^{k} \Phi(1) \in H^{k\{p\}}\left(X ; Z_{p}\right)
$$

Here $\Phi$ is the Thom isomorphism and $\wp^{k}$ is the $k$-th mod $p$ Steenrod power, and $\{p\}=1$ if $p=2$ and $\{p\}=2(p-1)$ if $p>2$. Note that, if $p=2, q_{k}(\xi)$ is the Stiefel-Whitney class of $\xi$.

If $p>2$ and $\xi$ is a vector bundle, we have the following characterization of the $\bmod p$ Wu classes ([8], pp. 227-230):

Theorem $\left[\mathbf{W u}\right.$. Let $\xi$ be a vector bundle and $u_{i}(\xi)$ its Pontrjagin classes reduced at an odd prime $p=2 s+1$. Then

$$
q_{k}(\xi)=K_{s k}\left(u_{1}(\xi), \cdots, u_{s k}(\xi)\right)
$$

where $\left\{K_{i}\right\}$ is the multiplicative sequence belonging to the power series $f(t)=$ $1+t^{s} \in Z_{p}[[t]]$

In particular, if $p=3$, then $s=1$ and it follows that $q_{k}(\xi)$ is equal to the Pontrjagin class $u_{k}(\xi)$ reduced mod 3 .

Now let us consider an $n$-dimensional $Z_{p}$-Poincaré complex $X$ with a prime integer $p$. Then, exploiting Poincaré duality, the class $v_{k}(X)=v_{k} \in H^{\{p\} k}\left(X ; Z_{p}\right)$ can be defined by the condition

$$
x \cup v_{k}=\wp^{k} x
$$

for any $x \in H^{n-k\{p\}}\left(X ; Z_{p}\right)$. We set $v(X)=v=1+v_{1}+v_{2}+\cdots$.

Let $\wp=1+\wp_{1}+\wp_{2}+\cdots$ be the mod $p$ total Steenrod power. Then we define the $\bmod p$ total Wu class $q(X)=q$ and the $k$-th Wu class $q_{k}$ by the equation

$$
\wp v=q(X)=1+q_{1}+\cdots, \quad q_{k} \in H^{\{p\} k}\left(X ; Z_{p}\right)
$$

Then the $\bmod p \mathrm{Wu}$ numbers are defined as $q_{I}(X)=\left\langle q_{\iota_{1}} q_{\iota_{2}} \cdots q_{\iota_{k}},[X]\right\rangle$ for any sequence of natural numbers $I=\left(\iota_{1}, \iota_{2}, \cdots, \iota_{k}\right)$ such that $\{p\}\left(\iota_{1}+\iota_{2}+\cdots+\iota_{k}\right)=n$. (When $p=2$, the classical terminology ([8]) calls $q_{k}$ the Stiefel-Whitney class of $X$ and $v_{k}(X)$ the Wu class of $X$. Our terminology is motivated by [9].)

Note that the classes $v_{k}(X)$ are defined in the above without referring to any specific sphere fibration. However, if $X$ is a Poincaré complex, a theorem of W. Browder ([2], p. 64) shows that $v(X) \cup U=\wp^{-1} U$, when $p=2$, where $U$ is the Thom class of the Spivak normal fibration $\xi$ of $X$ and $\wp^{-1}$ is the multiplicative inverse of $\wp$. The proof can easily be modified for any prime $p$. In particular, this theorem implies that $q_{k}(X)=q_{k}(\eta)$, where $\eta$ is the fibration stably inverse to $\xi$.

\section{VANishing of the MOD $p$ WU NUMBers}

Proof of Lemma 2. We may assume that $n \equiv 0 \bmod 2(p-1)$ if $p$ is an odd prime; otherwise the lemma is trivially true.

Claim. $\varphi \cup D q=0$. 
Let us assume the claim, which will be proved in a moment.

First of all, we observe that, for any even dimensional cohomology class $u$ such that $D(\varphi \cup u)=0$, we have: $u=(-1)^{r+1} \varphi \cup D u$. In particular, $\varphi \cup u=0$ implies $u=(-1)^{r+1} \varphi \cup D u$.

Let $I=\left(\iota_{1}, \iota_{2}, \cdots, \iota_{l}\right)$ be a sequence of natural numbers such that $\{p\}\left(\iota_{1}+\iota_{2}+\cdots+\iota_{l}\right)=n$. Set $q_{I}=q_{\iota_{1}} q_{\iota_{2}} \cdots q_{\iota_{l}}$. Then, $q_{I}=(-1)^{r+1} \varphi \cup D q_{I}=$ $(-1)^{r+1} \varphi \cup \sum_{j} D q_{\iota_{j}} q_{\iota_{1}} \cdots \widehat{q_{\iota_{j}}} \cdots q_{\iota_{l}}=(-1)^{r+1} \sum_{j}\left(\varphi \cup D q_{\iota_{j}}\right) q_{\iota_{1}} \cdots \widehat{q_{\iota_{j}}} \cdots q_{\iota_{l}}=0$ (the last equality follows from the claim).

Proof of the claim. Let $v_{k}$ be as defined in the previous section. Then

$$
\wp^{k} x=x \cup v_{k}
$$

for any $x$ such that $|x|=n-\{p\} k$. Note that $|x|$ is even if $p$ is odd, because $n \equiv 0$ $\bmod \{p\}$. Apply $D$ to the both sides of the equation and then multiply them by $\varphi$ to get

$$
\varphi \cup \wp^{k} D x=x \cup\left(\varphi \cup D v_{k}\right)+(\varphi \cup D x) \cup v_{k}
$$

Case 1. Assume $p=2$ or $r$ is even. Then, noting that $\wp \varphi=\varphi+\varphi^{p}$ from the hypothesis, we obtain, by Cartan's formula,

$$
\wp^{k}(\varphi \cup D x)=\sum_{i} \wp^{k-i} \varphi \cup \wp^{i} D x=\varphi \cup \wp^{k} D x+\varphi^{p} \cup \wp^{k-\kappa} D x,
$$

where $\kappa=r$ if $p=2$ and $\kappa=r / 2$ if $p>2$, and $\wp^{j} \equiv 0$ is understood if $j<0$. By the equations (1) and (2), we have

$$
\wp^{k}(\varphi \cup D x)-\varphi^{p} \cup \wp^{k-\kappa} D x=x \cup\left(\varphi \cup D v_{k}\right)+(\varphi \cup D x) \cup v_{k} .
$$

However, $\wp^{k}(\varphi \cup D x)=(\varphi \cup D x) \cup v_{k}$, by the defining property of $v_{k}$. Together with equation $(3)$, we have

$$
\varphi^{p} \cup \wp^{k-\kappa} D x=-x \cup\left(\varphi \cup D v_{k}\right) .
$$

Next note that $\varphi^{p} \cup \wp^{k-\kappa} x=0$ for dimensional reasons and that $D \varphi^{p}=p \varphi^{p-1}=0$. We therefore have

$$
0=D\left(\varphi^{p} \cup \wp^{k-\kappa} x\right)=D \varphi^{p} \cup \wp^{k-\kappa} x+\varphi^{p} \cup D \wp^{k-r} x=\varphi^{p} \cup \wp^{k-\kappa} D x .
$$

Plugging the extreme equation of (5) into equation (4), we get $\left(\varphi \cup D v_{k}\right) \cup x=0$ for any $x$ such that $|x|=n-\{p\} k$. By Poincaré duality, we conclude that $\varphi \cup D v_{k}=0$ and, therefore, that $\varphi \cup D v=0$.

Now we apply the mod $p$ total Steenrod power to each side of the equation $\varphi \cup D v=0$, to get $0=\wp(\varphi \cup D v)=\left(\varphi+\varphi^{p}\right) \cup \wp D v$

It follows that $\varphi \cup \wp D v=-\varphi^{p} \cup \wp D v=\cdots=(-1)^{N} \varphi^{p N} \cup \wp D v=0$, where $N$ is any integer large enough.

Note that $\wp D v=D \wp v=D q$. So we conclude $\varphi \cup D q=0$, as required.

Case 2. Assume both $p$ and $r$ are odd.

Then $\wp \varphi=\varphi$ from the hypothesis and, therefore, $\wp^{k}(\varphi \cup D x)=\varphi \cup \wp^{k} D x$. Together with equation (1), we obtain

$$
\wp^{k}(\varphi \cup D x)=x \cup\left(\varphi \cup D v_{k}\right)+(\varphi \cup D x) \cup v_{k} .
$$

Since $\wp^{k}(\varphi \cup D x)=(\varphi \cup D x) \cup v_{k}$ by the defining property of $v_{k}$, we conclude that 
$x \cup\left(\varphi \cup D v_{k}\right)=0$ for any $x$ such that $|x|=n-2(p-1) k$. Therefore, $\varphi \cup D v_{k}=0$ and $\varphi \cup D v=0$. So $0=\wp(\varphi \cup D v)=\varphi \cup D \wp v=\varphi \cup D q$. This proves Lemma 2 .

Remark. It is a well known fact that the projective planes $F P^{n}$ are boundaries if and only if $n$ is odd, where $F$ is the real, the complex, or the quaternion field. In fact, if $n$ is even, $F P^{n}$ is not a boundary since $\chi\left(F P^{n}\right) \not \equiv 0 \bmod 2$. For the same reason the Cayley projective plane does not bound.

If $n$ is odd, Lemma 2 provides a unified argument to prove $F P^{n}$ bounds: Note that $\left.H^{*}\left(F P^{n} ; Z_{2}\right)=Z_{2}[x] /<x^{n+1}\right\rangle$ with $|x|=1,2$, or 4 . The formal derivation $D\left(x^{k}\right)=\bar{k} x^{k-1}$ on $Z_{2}[x]$ gives rise to a well defined derivation on $\left.Z_{2}[x] /<x^{n+1}\right\rangle$ because $n$ is odd. By inspection, $S q^{k} D=D S q^{k}$ for any integer $k$. Furthermore, $D x=1$ and $S q x=x+x^{2}$. By Lemma 2, all the Stiefel-Whitney numbers of $F P^{n}$ vanish. Therefore $F P^{n}$ bounds.

Let $R$ be a commutative ring with a unit and $F: S^{r} \times X \rightarrow X$ be as in section 1. Then it can be shown that for any $u \in H^{*}(X ; R), F^{*}(u)=1 \times u+\overline{\left[S^{r}\right]} \times u^{\prime} \in$ $H^{*}\left(S^{r} \times X ; R\right)$ with a unique $u^{\prime} \in H^{*-r}(X ; R)$, where $\overline{\left[S^{r}\right]} \in H^{r}\left(S^{r} ; R\right)$ is the class such that $\left\langle\overline{\left[S^{r}\right]},\left[S^{r}\right]\right\rangle=1$. Then the derivation $D_{F}$ associated to $F$ is the group homomorphism $D_{F}: H^{*}(X ; R) \rightarrow H^{*-r}(X ; R)$, defined by $D_{F} u=u^{\prime}$.

Lemma 4 proves, in particular, that $D_{F}$ is a derivation of degree $-r$.

For simplicity of notation, we set $D=D_{F}$.

Lemma 4. (i) $D(u \cup v)=D u \cup v+(-1)^{r|u|} u \cup D v$ for any $u, v \in H^{*}(X ; R)$.

(ii) $D u=\left\langle u, f_{*}\left[S^{r}\right]\right\rangle 1$ for any $u \in H^{r}(X ; R)$, where $f$ is the map defined by $f(z)=F\left(z, x_{0}\right)$ and 1 the identity of the cohomology ring $H^{*}(X ; R)$.

(iii) If $R=Z_{p}$, we have $D \wp^{k}=\wp^{k} D$ for any integer $k$.

Proof. (i) We have $F^{*}(u \cup v)=1 \times(u \cup v)+\overline{\left[S^{r}\right]} \times D(u \cup v)$. On the other hand, $F^{*}(u \cup v)=F^{*}(u) \cup F^{*}(v)=\left(1 \times u+\overline{\left[S^{r}\right]} \times D u\right) \cup\left(1 \times v+\overline{\left[S^{r}\right]} \times D v\right)=$ $1 \times(u \cup v)+\overline{\left[S^{r}\right]} \times\left(D u \cup v+(-1)^{r|u|} u \cup D v\right)$.

Thus, $D(u \cup v)=D(u \cup v)+(-1)^{r|u|} u \cup D v$.

(ii) Let $\left[x_{0}\right]$ be the generator of $H_{0}(X)$ and $u \in H^{r}(X)$. Then, $\left\langle F^{*}(u),\left[S^{r}\right] \times\right.$ $\left.\left.\left[x_{0}\right]\right]\right\rangle=\left\langle 1 \times u+\overline{\left[S^{r}\right]} \times D u,\left[S^{r}\right] \times\left[x_{0}\right]\right\rangle=\left\langle\overline{\left[S^{r}\right]},\left[S^{r}\right]\right\rangle\left\langle D u,\left[x_{0}\right]\right\rangle=\left\langle D u,\left[x_{0}\right]\right\rangle$.

On the other hand,

$$
\left\langle F^{*}(u),\left[S^{r}\right] \times\left[x_{0}\right]\right\rangle=\left\langle u, F_{*}\left(\left[S^{r}\right] \times\left[x_{0}\right]\right)\right\rangle=\left\langle u, f_{*}\left[S^{r}\right]\right\rangle .
$$

Therefore, we conclude that $D u=\left\langle D u,\left[x_{0}\right]\right\rangle 1=\left\langle u, f_{*}\left[S^{r}\right]\right\rangle 1$.

(iii) Note that $\wp^{k} F^{*}(u)=F^{*} \wp^{k} u$. We have $\wp^{k} F^{*}(u)=\wp^{k}\left(1 \times u+\overline{\left[S^{r}\right]} \times D u\right)=$ $1 \times\left(\wp^{k} u\right)+\overline{\left[S^{r}\right]} \times\left(\wp^{k} D u\right)$ and $F^{*}\left(\wp^{k} u\right)=1 \times\left(\wp^{k} u\right)+\overline{\left[S^{r}\right]} \times\left(D \wp^{k} u\right)$.

Hence, $\wp^{k} D u=D \wp^{k} u$. This completes the proof of Lemma 5 .

Lemmas 2 and 4 together prove

Theorem 5. Let $p$ be a prime and $X$ a path connected $Z_{p}$-Poincaré space with a base point $x_{0}$. Assume there is $f: S^{r} \rightarrow X$ which represents an element of $G_{r}\left(X, x_{0}\right)$ such that there is a class $\varphi \in H^{r}\left(X ; Z_{p}\right)$ satisfying $\left\langle\varphi, f_{*}\left[S^{r}\right]\right\rangle=1$ and $\wp^{i} \varphi=0$ for any $i$ such that $0<i<[r / 2]$ if $p>2$ and $0<i<r$ if $p=2$. Then all the $\bmod p$ Wu numbers of $X$ vanish.

Note that, if we concentrate on the first evaluation subgroup $G_{1}\left(X, x_{0}\right)$, Theorem 5 takes a much simpler form: 
Corollary 6. Let $X$ be a path connected $Z_{p}$-Poincaré space with a base point $x_{0}$. If there is an element $\alpha \in G_{1}\left(X, x_{0}\right)$ whose image under the Hurewicz map $h$ : $\pi_{1}\left(X, x_{0}\right) \rightarrow H_{1}\left(X ; Z_{p}\right)$ is not zero, then all the mod $p$ Wu numbers vanish.

Proof. In view of Theorem 5, it is enough to show the existence of $\varphi \in H^{1}\left(X ; Z_{p}\right)$ such that $\langle\varphi, h(\alpha)\rangle=1$. But the map from $H^{1}\left(X ; Z_{p}\right)$ into $\operatorname{Hom}\left(H_{1}(X), Z_{p}\right)$ defined by the Kronecker index is an isomorphism, which completes the proof.

The following lemma is due to D. H. Gottlieb ([3]).

Lemma 7. Let $X$ be an aspherical complex. If $f: S^{1} \rightarrow X$ represents a central element in $\pi_{1}\left(X, x_{0}\right)$, then $f$ represents an element of the first evaluation subgroup.

Proof. Consider $f$ as a map from $(I,\{0,1\})$ into $\left(X, x_{0}\right)$. We may also assume $x_{0} \in X$ is a nondegenerate base point. Then $1_{X} \cup f:\{0\} \times X \cup I \times\left\{x_{0}\right\} \rightarrow X$ extends to a map $F: I \times X \rightarrow X$.

Set $g=\left.F\right|_{\{1\} \times X}$. Then $g$ preserves the base point and $g_{\sharp}(\alpha)=[f]^{-1} \alpha[f]$ for any $\alpha \in \pi_{1}\left(X, x_{0}\right)$. However, $[f]$ is central. So $g_{\sharp}$ is the identity homomorphism of $\pi_{1}\left(X, x_{0}\right)$. Since $X$ is an aspherical complex, $g$ is homotopic to the identity by a base point preserving homotopy ([12], Theorem 9, pp. 424-432). Hence there is no loss of generality in assuming $g=\left.F\right|_{\{1\} \times X}$ is the identity map. Thus $F$ can be considered as a map from $S^{1} \times X$ into $X$ which satisfies $F\left(z, x_{0}\right)=f(z)$ and $F\left(z_{0}, x\right)=x$ for all $x \in X$ for some base point $z_{0} \in S^{1}$.

Finally, Corollary 6 and Lemma 7 together prove Theorem 1.

\section{The Poincaré complexes}

Let $(Y, X)$ be a Poincaré pair in the sense of C.T.C. Wall ([15]). Note, in particular, that both $Y$ and $X$ are finitely dominated complexes, and are allowed to be infinite. Of course, we do not exclude the case $X=\phi$ and will identify $(Y, \phi)$ with $Y$ for any topological space $Y$. We shall say $Y$ is a Poincaré complex if and only if $(Y, \phi)$ is a Poincaré pair.

For any Poincaré pair $(Y, X)$ of formal dimension $n$, if $k$ is a sufficiently large integer, there exists a fibration $\nu^{k}$ over $Y$, called the Spivak fibration of $(Y, X)$, such that its fiber is $S^{k-1}$ and there is a degree one map $\psi: S^{n+k} \rightarrow T(\nu) / T\left(\left.\nu\right|_{X}\right)$. Here $T(\xi)$ means the Thom space of $\xi$ for any spherical fibration $\xi$, and $\psi$ is a degree one map in the sense it induces an isomorphism between the top dimensional homology groups with integer coefficients. The Spivak fibration of a Poincaré pair is welldefined up to stable fiber homotopy equivalence: any two spherical fibrations with degree one map over a Poincaré pair are stably fiber homotopy equivalent to each other by a stable fiber homotopy equivalence preserving the degree one maps ([13] and also [15]).

Now we shall introduce the notion of Poincaré embedding, essentially following Wall's definition in [14], p. 113.

Let $M^{m}, V^{m+q}$ be Poincaré complexes and $\iota: M \rightarrow V$ be a continuous map. Then the realization of $\iota$ as a Poincaré embedding shall consist of:

a $(q-1)$-spherical fibration $\nu_{\iota}$ with projection $p: E \rightarrow M$,

a Poincaré pair $(C, E)$, and 
a homotopy equivalence $h: C \cup M(p) \rightarrow V$, where $M(p)$ is the mapping cylinder of $p$ and $C \cap M(p)=E$, so that the following diagram commutes up to homotopy:

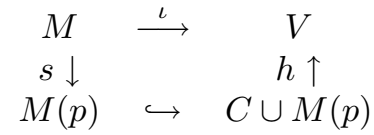

where $s$ is the natural inclusion.

We shall call $\nu_{\iota}$ in the above a normal fibration of $\iota$.

From now on, for any Poincaré pair $(Y, X), \nu_{Y}$ will denote the stable Spivak fibration of $(Y, X)$. The following statement is due to C.T.C. Wall ([14], p. 115).

Lemma 8. Let $M$ and $V$ be Poincaré complexes. Assume $\iota: M \rightarrow V$ is a continuous map which admits a realization as a Poincaré embedding with normal fibration $\nu_{\iota}$. Then,

$$
\nu_{M}=\nu_{\iota}+\iota^{*} \nu_{V} .
$$

Remark. Lemma 8 implies that the normal fibration of a continuous map is unique up to stable fiber homotopy equivalence.

Consider a fibration $F^{m} \stackrel{i}{\rightarrow} Y^{m+n} \stackrel{f}{\rightarrow} X^{n}$, in which $F, Y, X$ are connected Poincaré complexes of formal dimensions $m, m+n, n$, respectively. Let $C \cup M(p)=$ $X$ be a realization of the inclusion $\iota: M^{n-k} \hookrightarrow X^{n}$ as a Poincaré embedding with a normal fibration $\nu_{\iota}$ whose projection is $p: E \rightarrow M$. Here we identify $M$ with a subspace of $M(p)$ by the natural inclusion $s: M \hookrightarrow M(p)$ as in the above. Note that $f^{-1} M$ is a Poincaré complex by a theorem of Gottlieb ([6]) (even if Gottlieb proves the theorem for the fibration sequence of spaces which are homotopic to finite complexes, his proof can be extended to general Poincaré complexes, exploiting a result of Mather ([7]) that $X$ is a finitely dominated complex if and only if $X \times S^{1}$ is homotopic to a finite complex together with a theorem of Wall ([15]) that $A \times B$ is a Poincaré complex if and only if $A$ and $B$ are).

Let $\hat{p}$ be the composite $f^{-1} E \hookrightarrow f^{-1} M(p) \simeq f^{-1} M$ and consider the following diagram which commutes up to homotopy:

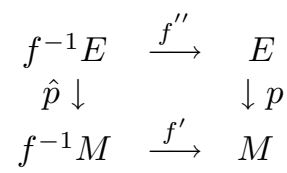

where $f^{\prime}, f^{\prime \prime}$ are $f$ restricted at the corresponding subspaces. Since the homotopy fibers of $f^{\prime}$ and $f^{\prime \prime}$ are both $F$, the above diagram is a homotopy pull-back square. Therefore, $\hat{p}$ has the same homotopy fiber as $p$, that is, $S^{k-1}$. Thus we conclude that $\hat{p}$ is the projection of the spherical fibration $f^{\prime *} \nu_{\iota}$ up to homotopy. We also assume $\left(f^{-1} C, f^{-1} E\right)$ is a Poincaré pair (which is redundant if $k \geq 3$ by Wall [15], Lemma 11.1, but, even when $k \leq 2$, we still conjecture the assumption is not necessary, presuming a relative version of the theorem by Gottlieb mentioned above holds). These data give a realization of the inclusion $\bar{\iota}: f^{-1} M \hookrightarrow Y$ as a Poincaré embedding with normal fibration $f^{\prime *} \nu_{\iota}$. And it follows that the Spivak fibration of $f^{-1} M$ is $f^{\prime *} \nu_{\iota}+\bar{\iota}^{*} \nu_{Y}$ by Lemma 8. In particular, we have:

Theorem 9. Let $F^{m} \stackrel{\iota}{\rightarrow} Y^{n+m} \stackrel{f}{\rightarrow} X^{n}$ be a fibration sequence in which $F, Y, X$ are connected Poincaré complexes of formal dimension $m, n+m, n$, respectively. Furthermore, assume $X=D^{n} \cup K, D^{n} \cap K=S^{n-1}$, and $\left(K, S^{n-1}\right),\left(f^{-1} K, f^{-1} S^{n-1}\right)$ 
are Poincaré pairs. Then

$$
\nu_{F}=\iota^{*} \nu_{Y}
$$

Remark. If $n \geq 3$, the conditions of Theorem 9 to ensure that the inclusions of a point and the fiber into $X$ and $Y$ respectively can be realized as Poincaré embeddings in a consistent way are redundant by results of Wall ([14], Theorem 2.4. and [15], Lemma 11.1).

Theorem 10 is a consequence of a theorem by Gottlieb ([5]) if the projection is a smooth map. However, his technique can be applied directly to a corresponding homotopy theoretic situation. In the rest of this paper, the homology groups are the usual ones with a commutative ring with identity as their coefficients. Furthermore, $[A](\overline{[A]})$ will denote a generator of the top dimensional homology (cohomology) for any space $A$, and we define $[A, B](\overline{[A, B]})$ similarly for any pair $(A, B)$.

Theorem 10 (D. Gottlieb). Let $F^{m} \stackrel{\iota}{\rightarrow} Y^{n+m} \stackrel{f}{\rightarrow} X^{n}$ be a fibration sequence in which $F, Y, X$ are connected Poincaré complexes of formal dimension $m, n+m$, $n$, respectively. Assume $X=D^{n} \cup K$, where $D^{n} \cap K=S^{n-1}$ and $\left(K, S^{n-1}\right)$, $\left(f^{-1} K, f^{-1} S^{n-1}\right)$ are Poincaré pairs. Then

$$
\iota_{*}[F]=f^{*} \overline{[X]} \cap[Y] .
$$

Proof. We introduce the following two commutative diagrams:

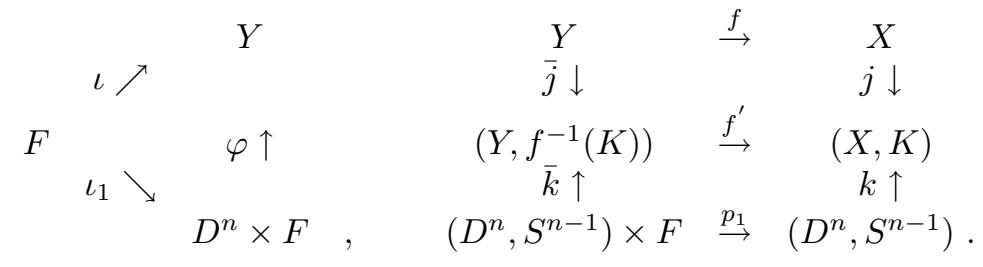

In the first diagram, $\varphi$ is a fiber preserving map which restricts to a homotopy equivalence at each fiber, and $\iota, \iota_{1}$ are the inclusions defined by a base point $x_{0}$. In the second diagram, $f^{\prime}$ is the map defined by $f, p_{1}$ is the projection, and all the vertical maps are inclusions except for $\bar{k}$ which is defined by $\varphi$. Note that the vertical maps in the second diagram induce isomorphisms between the top dimensional homology groups. In particular, to prove $j$ and $\bar{j}$ are degree one maps, we exploit the assumption that $\left(K, S^{n-1}\right)$ and $\left(f^{-1} K, f^{-1} S^{n-1}\right)$ are Poincaré pairs, respectively.

In the calculations to follow, we shall repeatedly use the following, which we call rule* $\left([12]\right.$, p. 254): Let $A_{1}, A_{2} \subset X$ and $B_{1}, B_{2} \subset Y$, and let $g: X \rightarrow Y$ map $A_{i}$ to $B_{i}, i=1,2$. Let $g_{i}:\left(X, A_{i}\right) \rightarrow\left(Y, B_{i}\right), i=1,2$, and $\bar{g}:\left(X, A_{1} \cup A_{2}\right) \rightarrow\left(Y, B_{1} \cup B_{2}\right)$ be the maps defined by $g$. Then, for any $u \in H^{q}\left(Y, B_{1}\right)$ and $z \in H_{n}\left(X, A_{1} \cup A_{2}\right)$, we have

$$
g_{2 *}\left(g_{1}^{*} u \cap z\right)=u \cap \bar{g}_{*} z \in H_{n-q}\left(Y, B_{2}\right)
$$

First of all, we observe that

$$
f^{*} \overline{[X]}=\bar{j}^{*} f^{\prime *} \overline{[X, K]} \in H^{m+n}(Y) .
$$

Therefore, we have

$$
\begin{aligned}
f^{*} \overline{[X]} \cap[Y] & =\bar{j}^{*} f^{\prime *} \overline{[X, K]} \cap[Y] \\
& =f^{\prime *} \overline{[X, K]} \cap \bar{j}_{*}[Y] \\
& =f^{\prime *} \overline{[X, K]} \cap\left[Y, f^{-1} K\right] \in H_{m}(Y)
\end{aligned}
$$


in which we apply rule* above with $g_{1}=\bar{g}=\bar{j}$ and $g_{2}=1_{Y}$.

Furthermore, noting $\bar{k}$ is defined by $\varphi$, we have

$$
\begin{aligned}
\varphi_{*}\left(p_{1}^{*}\right. & \left.\overline{\left[D^{n}, S^{n-1}\right]} \cap\left(\left[D^{n}, S^{n-1}\right] \times[F]\right)\right) \\
& =\varphi_{*}\left(\bar{k}^{*} f^{\prime *} \overline{[X, K]} \cap\left(\left[D^{n}, S^{n-1}\right] \times[F]\right)\right) \\
& =f^{\prime *} \overline{[X, K]} \cap \bar{k}_{*}\left(\left(\left[D^{n}, S^{n-1}\right] \times[F]\right)\right. \\
& =f^{\prime *} \overline{[X, K]} \cap\left[Y, f^{-1} K\right] \in H_{m}(Y),
\end{aligned}
$$

in which, for the second equality, again we use rule*.

On the other hand,

$$
\varphi_{*}\left(\left(p_{1}^{*} \overline{\left[D^{n}, S^{n-1}\right]}\right) \cap\left(\left[D^{n}, S^{n-1}\right] \times[F]\right)\right)=\varphi_{*}\left(\iota_{1 *}[F]\right)=\iota_{*}[F]
$$

To summarize,

$$
\begin{aligned}
i_{*}[F] & =\varphi_{*}\left(p_{1}^{*} \overline{\left[D^{n}, S^{n-1}\right]} \cap\left(\left[D^{n}, S^{n-1}\right] \times[F]\right)\right) \\
& =f^{*}\left[\overline{[X, K]} \cap\left[Y, f^{-1} K\right]\right. \\
& =f^{*} \overline{[X]} \cap[Y] \in H_{m}(Y)
\end{aligned}
$$

which proves the theorem.

We will need the following ([10], Lemma 2).

Lemma 11 (V. Puppe). Consider the following diagram which commutes up to homotopy:

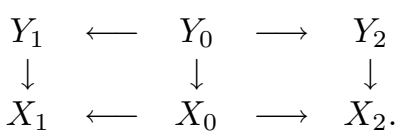

Assume that the maps at the top row of the above diagram induce homotopy equivalences between the homotopy fibers. Then, the homotopy fiber of the map from hocolim $\left(Y_{1} \leftarrow Y_{0} \rightarrow Y_{2}\right)$ into hocolim $\left(X_{1} \leftarrow X_{0} \rightarrow X_{2}\right)$ defined by the above diagram is the same homotopy type as those of $Y_{i} \rightarrow X_{i}, i=0,1,2$, and the natural maps from $Y_{i}$ 's to hocolim $\left(Y_{1} \leftarrow Y_{0} \rightarrow Y_{2}\right)$ induce the homotopy equivalences between the homotopy fibers.

Definition. Given a spherical fibration $\xi$ over a complex $X$, let $\bar{\xi}: X \rightarrow B G$ be the continuous map classifying $\xi$ up to stable fiber homotopy equivalence. If $R$ is a commutative ring with identity, for any $\alpha \in H^{i}(B G ; R)$, we shall call $\bar{\xi}^{*} \alpha \in$ $H^{i}(X ; R)$ the $(\alpha-)$ characteristic class coming from $\xi$.

In particular, if $i=n$ and $X$ is a Poincaré complex of formal dimension $n$, oriented with respect to the coefficient $R$, that is, $H_{n}(X ; R)$ is isomorphic to $R$ with a preferred generator $[X] \in H_{n}(X ; R), \bar{\xi}^{*} \alpha$ will be called the $(\alpha-)$ characteristic number, identifying it with $\left\langle\bar{\xi}^{*} \alpha,[X]\right\rangle \in R$.

If $X$ is a Poincaré complex of formal dimension $n$, oriented with respect to the coefficient $R$ and $\nu$, the Spivak normal fibration over $X$, then $\nu$ gives rise to the characteristic numbers as in the above. Note that, by the naturality of the cup product and the Steenrod squares, the equality $v(X) \cup U=\wp^{-1} U$ in the last paragraph of section 2 implies that the classes $v_{k}(X) \in H^{k}\left(X ; Z_{p}\right)$ and, therefore, the $q_{k}(X)$ 's are the characteristic classes coming from the Spivak normal fibration. Therefore, Theorem 3 implies vanishing of all the $\bmod p \mathrm{Wu}$ numbers. 
Proof of Theorem 3. Let $z_{0}, x_{0}$ be the base points of $S^{k}, M$, respectively. We choose a map $F: S^{k} \times M \rightarrow M$ such that $F\left(z_{0}, \cdot\right): M \rightarrow M$ is the identity and $F\left(\cdot, x_{0}\right)$ : $S^{k} \rightarrow M$ represents $\alpha$. We shall denote the $(k+1)$-dimensional upper and lower hemispheres by $D_{+}^{k+1}, D_{-}^{k+1}$, respectively. Then we define $F^{\prime}: S^{k} \times M \rightarrow S^{k} \times M$ by the rule $F^{\prime}(z, x)=(z, F(z, x))$ and, subsequently, $\bar{F}$ as the composite $S^{k} \times M \stackrel{F^{\prime}}{\rightarrow}$ $S^{k} \times M \hookrightarrow D_{+}^{k+1} \times M$.

The following diagram commutes:

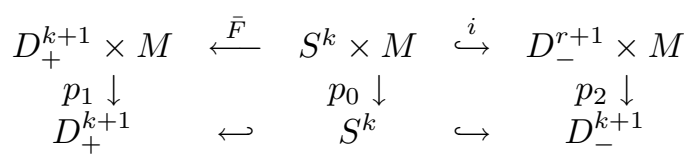

in which $p_{i}, i=0,1,2$, are the projections and the hooked arrows are inclusions. Let $E$ be the push-out of the top row and $p: E \rightarrow S^{k+1}$ be the push-out of $p_{1} \leftarrow p_{0} \hookrightarrow p_{2}$. Note that $E$ is in fact the homotopy push-out, for the inclusions on the right hand side are cofibrations.

The vertical arrows are bundle projections with fiber $M$ and the horizontal arrows in the top row preserve the fibers. In particular, $\bar{F}$ restricts to a homotopy equivalence at each fiber. Therefore, by Lemma 11, we obtain a fibration sequence

$$
M \stackrel{\iota}{\rightarrow} E \stackrel{p}{\rightarrow} S^{k+1}
$$

up to homotopy. Since the fiber and the base space are Poincaré complexes, $E$ is also a Poincaré complex by the same argument we used in the discussion to derive Theorem 9.

Clearly, the assumptions of Theorem 9 are satisfied for the fibration $M \stackrel{\iota}{\rightarrow} E \stackrel{p}{\rightarrow}$ $S^{k+1}$. Thus the Spivak fibration of $M$ is the pull-back of the Spivak fibration of $E$ by $\iota$, and therefore

$$
\bar{\nu}_{M}=\bar{\nu}_{E} \iota,
$$

where $\bar{\nu}_{M}, \bar{\nu}_{E}$ are the classifying maps of the Spivak fibrations of $M, E$, respectively, into $B G$.

Therefore, to complete the proof of Theorem 3, it is enough to show:

Claim. With a field of coefficients $R, \iota^{*}: H^{n}(E) \rightarrow H^{n}(M)$ is the null homomorphism.

Proof. We may assume $M \stackrel{\iota}{\rightarrow} E \stackrel{p}{\rightarrow} S^{k+1}$ is already a fibration.

Consider the following portion of the exact sequence (see [12], sec. 8.5):

$$
H_{k+1}(M) \stackrel{\iota *}{\rightarrow} H_{k+1}(E) \stackrel{j}{\rightarrow} H_{k+1}\left(\left(D_{-}^{k+1}, S^{k}\right) \times M\right) \stackrel{F_{*} \partial}{\rightarrow} H_{k}(M),
$$

which we shall call exact sequence* . Note that $\partial: H_{k+1}\left(\left(D_{-}^{k+1}, S^{k}\right) \times M\right) \rightarrow$ $H_{k}\left(S^{k} \times M\right)$ is an isomorphism. Furthermore, consider the commutative diagram

$$
\begin{array}{ccc}
S_{\uparrow}^{k} \times M & \stackrel{F}{\rightarrow} & M \\
S^{k} \times\left\{x_{0}\right\} & \nearrow \bar{\alpha} & \\
&
\end{array}
$$

where $\bar{\alpha}: S^{k}=S^{k} \times\left\{x_{0}\right\} \rightarrow M$ is the map defined by $\bar{\alpha}(z)=F\left(z, x_{0}\right)$, which represents $\alpha$. Thus, the image by $F_{*} \partial: H_{k+1}\left(\left(D_{+}^{k+1}, S^{k}\right) \times M\right) \rightarrow H_{k}(M)$ cannot be trivial since it must contain $h(\alpha) \neq 0$. 
However, $H_{k+1}\left(\left(D_{-}^{k+1}, S^{k}\right) \times M\right)$ is a vector space with one generator over $R$. Therefore, $F_{*} \partial$ is a monomorphism and $j$ in exact sequence* is the zero homomorphism.

Now we introduce the following commutative diagram:

$$
\begin{array}{ccccc}
H_{k+1}(E) & \stackrel{\bar{q}}{\rightarrow} & H_{k+1}\left(E, D_{+}^{k+1} \times M\right) & \stackrel{\overline{e x c}}{\leftarrow} & H_{k+1}\left(\left(D_{-}^{k+1}, S^{k}\right) \times M\right) \\
p_{*} \downarrow & & p_{*}^{\prime} \downarrow & & p_{1 *} \downarrow \\
H_{k+1}\left(S^{k+1}\right) & \stackrel{q}{\rightarrow} & H_{k+1}\left(S^{k+1}, D_{+}^{k+1}\right) & \stackrel{e x c}{\rightleftarrows} & H_{k+1}\left(D_{-}^{k+1}, S^{k}\right),
\end{array}
$$

where all the horizontal arrows are induced by inclusions, and the vertical arrows by restrictions of the projection $p: E \rightarrow S^{k+1}$. By inspection, we know that all the arrows are isomorphisms except for, possibly, $\bar{q}$ and $p_{*}$. Note that the homomorphism $j$ in exact sequence* is none other than $\overline{e x c}^{-1} \bar{q}$. Therefore, $p_{*}=$ $q^{-1}$ exc $p_{1_{*}} j=0$. By a universal coefficient theorem, $p^{*}: H^{k+1}\left(S^{k+1}\right) \rightarrow H^{k+1}(E)$ is also the the null homomorphism. However, $\iota_{*}[M]=p^{*}\left[S^{k+1}\right] \cap[E]$ by Theorem 10. Therefore, $\iota_{*}=0$ on $H_{n}(M)$ and it follows that $\iota^{*}=0$ on $H^{n}(E)$, which completes the proof of the claim.

\section{REFERENCES}

[1] M. F. Atiyah and F. Hirzebruch, Cohomologie-Operationen und characteristische Klassen, Math. Zeitschr. 77 (1961), pp. 149-187. MR 27:6285

[2] W. Browder, Surgery on Simply-Connected Manifolds, Ergebnisse der Mathematik und Ihrer Grenzgebiete Band 65, Springer-Verlag, Berlin, 1972, pp.60-64. MR 50:11272

[3] D. H. Gottlieb, A Certain Subgroup of the Fundamental Group, Amer. J. Math. 87 (1965), pp. 840-856. MR 32:6454

[4] D.H. Gottlieb, Evaluation Subgroups of Homotopy Groups, Amer. J. Math. 91 (1969), pp. 729-756. MR 43:1181

[5] D.H. Gottlieb, Partial Transfers in Geometric Applications of Homotopy Theory I. Lecture Notes in Mathematics, no. 657, Springer Verlag, 1978, pp. 255-266. MR 80h:57024

[6] D.H. Gottlieb Poincaré Duality and Fibrations, Proceedings of the American Mathematical Society, 76. (1979), pp. 148-150. MR 81a:57017

[7] M. Mather, Counting Homotopy Types of Manifolds, Topology, 4 (1965), pp. 93-94. MR 31:742

[8] J. W. Milnor and J. D. Stasheff, Characteristic Classes, Annals of Mathematics Studies, no. 76, Princeton University Press (1974), pp. 115-137, 227-230. MR 55:13428

[9] J. W. Milnor, On Characteristic Classes for Spherical Fibre Spaces, Comm. Math. Helv. (1968), pp. 51-77. MR 37:2227

[10] V. Puppe, A Remark on "Homotopy Fibration", Manuscripta Math. 12 (1974), pp. 113-120. MR 51:1808

[11] N. E. Steenrod and D. B. A. Epstein, Cohomology Operations, Annals of Mathematics Studies no. 50, Princeton University Press (1962). MR 26:3056

[12] E. H. Spanier, Algebraic Topology, McGraw-Hill, (1966), pp. 254, 286-292, 424-432, 455. MR 35:1007

[13] M. Spivak, Spaces Satisfying Poincaré Duality, Topology, 6 (1967), pp. 77-101. MR 35:4923

[14] C.T.C. Wall, Surgery on Compact Manifold, Academic Press (1970). MR 55:4217

[15] C.T.C. Wall, Poincaré Complexes I. Annals of Mathematics, 86 (1967), pp. 213-245. MR 36: 880

Department of Mathematics, University of Notre Dame, Notre Dame, Indiana 465565683

Current address: Department of Mathematics, Hanyang University, Seoul, 133-791 Korea

E-mail address: Yanghyun.Byun.1@nd.edu 